\title{
Robust Control Design using Discrete Sliding Mode Control for Higher-Order Uncertain Systems
}

\author{
Y. P. Patil, H. G. Patel
}

\begin{abstract}
This paper considers a tracking problem on discrete-time higher-order linear time-delay systems. The improved observer-model following sliding mode controller (OMF-SMC) is proposed. The combination uses a classical Luyenberger observer based controller to achieve predefined process output and sliding mode controller is added to assure the robustness despite of uncertainty and external disturbances. To show the effectiveness of proposed method, four error performance indices, maximum peak overshoot and settling time are considered rigorously. The simulations results on the non-oscillatory, moderate oscillatory, integrating, unstable and non-minimum phase system demonstrates that the proposed approach performs better compared with classical PID controller, continuous and discrete sliding mode controllers.
\end{abstract}

Keywords: Discrete-time sliding-mode control, higher order delay time process, observer, model following control.

\section{INTRODUCTION}

$S_{1}$ liding mode control (SMC) is one of the popular robust control approach in control system engineering and technology. The basic SMC strategy is well-known to the world by Utkin [1]. In SMC design approach two steps are used, first is the selection of desired sliding surface and second is to estimate/evaluate control law based on system equations and selected surface. SMC is a robust controller design method used for regulation or/and tracking problems over conventional Proportional-Integral-Derivative (PID) [2], the Model Predictive Controller (MPC), Model-Based Controllers (MBC). SMC method can be implemented in the continuous and/or discrete time domain but due to the increasing demand of digitization, Discrete-Time Sliding Mode Controller design (DTSMC) approach is preferable [3]. Robust controller design for small order and higher order linear and non-linear plants along with perturbation and external disturbances is most common interest shown by researchers based on DTSMC approach [4]-[6]. Higher order

Revised Manuscript Received on February 05, 2020.

* Correspondence Author

Y. P. Patil*, Department of Instrumentation and Control, MVPS's KBT College of Engineering, Nashik, Affiliated to SPPU, Pune, India. Email: patil.yogesh@kbtcoe.org

H. G. Patel, Department of Electrical Engineering, Sardar Vallabhbhai National Institute of Technology, Surat, India. Email: hpatel.svnit@gmail.com

(C) The Authors. Published by Blue Eyes Intelligence Engineering and Sciences Publication (BEIESP). This is an open access article under the CC BY-NC-ND license (http://creativecommons.org/licenses/by-nc-nd/4.0/)
SMC design strategy is also a special research interest by others. The Zhong and Rees [7] proposed a non-SMC based Model Following Control (MFC) for a linear system without handling uncertainties in the input matrix. This limitation can be overcome by Phadke and Talole [8] by SMC design based MFC and considering the uncertainty and disturbances without knowledge of bounds. SMC design for uncertainty and disturbance estimation (UDE) for non-linear systems using MFC is reported by Deshpande and Phadke [9]. Inertial delay control (IDC) based SMC design for vehicle suspension to follow a modified skyhook model reported in [10]. Recently Suryawanshi et al. [11] reported robust SMC based on MFC strategy for an uncertain nonlinear system subjected to time-varying disturbance and application to an inverted pendulum system.

Observer-based DTSMC techniques for different applications are the interest of many researchers. Many approaches implemented for sensorless rotor speed observer of induction machines such as in [12] is common. Recently, in [13], [14] DTSMC is designed for sensor-less vector control of permanent magnet synchronous machine, and sensorless control on induction motor drives is implemented. In [15], chattering-free digital sliding mode control with state observer and disturbance rejection is presented and in [16], the design of estimator-based sliding-mode output-feedback controllers for discrete-time systems is simulated. In [17], an observer-based sliding mode control (OBSMC) problem is investigated for a class of uncertain delta operator systems with nonlinear exogenous disturbance. Also, in [18], a supertwisting algorithm for nonlinear systems with a state observer is discussed. Recently, several robust control approaches have been proposed to attenuate the undesired effects caused by the disturbances, uncertainties or the nonlinearities. Various methods applied in practical control systems, such as terminal sliding mode control [19], [20], adaptive super twisting terminal sliding mode control [21], [22], Fast terminal sliding mode control (FTSMC) [23], Nonsingular FTSMC [24], Adaptive nonsingular FTSMC [25], [26], [27], Continuous nonsingular FTSMC [28], Finite-time adaptive integral backstepping FTSMC [29].

The observer-model following design objective is to develop a control scheme which forces the plant-observer dynamics to follow the dynamics of a reference (ideal) model. To handle three major problems concerning linear optimal control techniques arises because of difficulties in specifying design objectives in terms of a performance index, large variations in plant parameters in the presence of disturbances and also unavailability of all states for measurement due to sensor constraints practically.

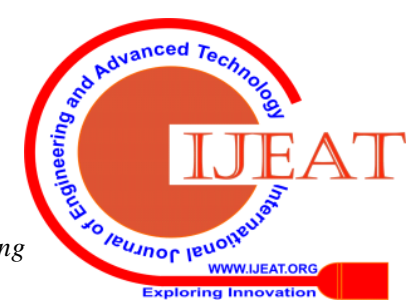


This motivates to use model-observer states in designing sliding mode control law. The reference model can be selected such that it specifies all the required design objectives. The robust SMC design handles the problem of uncertainty and disturbances (UAD). The OMF controller tends to force the plant-observer and model states to zero as time tends to infinity. This ensure the plant-observer output follows the model output accurately [30].

In this paper, a simple control approach proposed based on conventional Luyenberger observer [31] and model following control technique (OMF) for robust tracking of uncertain higher order time-delay systems. The sliding surface [32], which guarantees that the error between the model and plant states tends to zero within finite time. This approach does not require any global convergence properties and so it is well suited to uncertain time-delay plants.

The organization of the paper is as follows: Section 2 describes problem formulation. Section 3, introduces proposed discrete time OMF-SMC design procedures. Section 4, proves Lyapunov stability analysis. Subsequently, Section 5 rigorously discusses five examples to verify the applicability of proposed novel control. Concluding remarks are in Section 6.

\section{OVERVIEW OF OBSERVER-MODEL FOLLOWING APPROACH}

\section{A. Problem Formulation}

A model-following problem is considered with unavailability of states for measurement. Consider a continuous nominal linear time-invariant (LTI) single input single output (SISO) system [30]:

$$
\begin{gathered}
\dot{x}=A x+b u+\Delta A x+\Delta b u+d(x, t) . \\
y=C x
\end{gathered}
$$

where $x, u$ are the state vector and control input respectively. $A$ and $b$ are known constant matrices, $\Delta A, \Delta b$ are uncertainties and $d(x, t)$ is unknown disturbance. The terms $\Delta A, \Delta b$ and $d(x, t)$ satisfy the matching conditions [30]:

$$
\Delta A=b D, \quad \Delta b=b E, \quad d(x, t)=b v(x, t)
$$

where $D$ and $E$ are unknown matrices of appropriate dimensions and $v(x, t)$ is an unknown function. The system (1) can be written as:

$$
\dot{x}=A x+b u+b e(x, t)
$$

where $e(x, t)=D x+E u+v(x, t)$, the term $e(x, t)$ contains uncertainty and disturbance now referred to as lumped uncertainty. The following assumptions are consider for the control design:

1) The pair $(A, b)$ is controllable and the pair $(A, C)$ is observable.

2) Controllability and observability are not lost upon sampling.

3) The disturbance $f(x, t) \equiv f(t)$ is smooth and uniformly bounded.

Let the reference model is defined as,

$$
\dot{x}_{m}=A_{m} x_{m}+b_{m} u_{m}
$$

The choice of a model is such that:

$$
A-A_{m}=b G, \quad b_{m}=b M
$$

where $G$ and $M$ are suitable known matrices.

The objective is to design a control $u$ so as to force the plant (3) to follow the model (4) in spite of the parameter variations. The (2) and (5) are well-known matching conditions required to guarantee invariance and are explicit statements of the structural constraints stated in [33]. Proceeding further, the discretized counterpart of (1) is,

$$
\begin{gathered}
x(k+1)=\phi x(k)+\Delta \phi x(k)+\Gamma u(k)+\Delta \Gamma u(k) \\
+d_{d}(k),=x(0) \\
y(k)=C x(k)
\end{gathered}
$$

where

$$
\begin{gathered}
\phi=e^{A T}, \quad \Gamma=\int_{0}^{T} e^{A T} d \tau B \\
d_{d}(k)=\int_{0}^{T} e^{A T} B f((K+1) T-\tau) d \tau .
\end{gathered}
$$

where $\mathrm{T}$ is the sampling period. Here the disturbance $d(k)$ represents the influence accumulated from $k T$ to $(k+1) T$ , in the sequel it shall directly link to $x(k+1)=x((k+$ 1) $T$ ). From the definition of $\Gamma$ it can be shown that

$$
\begin{gathered}
\Gamma=\mathrm{BT}+\frac{1}{2 !} A B T^{2}+\cdots=B T+M T^{2}+O\left(T^{3}\right) \Rightarrow B T \\
=\Gamma-M T^{2}+O\left(T^{3}\right),
\end{gathered}
$$

where $M$ is a constant matrix because $T$ is fixed.

The uncertainties, $\Delta \phi, \Delta \Gamma$ and disturbance $d_{d}(k)$ satisfy the matching conditions given by the system:

$$
\Delta \phi=\Gamma D_{d}, \quad \Delta \Gamma=\Gamma E_{d}, \quad d_{d}(k)=\Gamma v_{d}(k)
$$

where $D_{d}$ and $E_{d}$ are unknown matrices of appropriate dimensions and $v_{d}(k)$ is an unknown function.

The system (6) can be written as:

$$
x(k+1)=\phi x(k)+\Gamma u(k)+e(k)
$$

where $e(k)=\Delta \phi x(k)+\Delta \Gamma u(k)+d_{d}(k)$ the term, $e(k)$ referred as lumped uncertainty as in linear domain.

The discretised part of the reference model is also written as:

$$
\begin{gathered}
x_{m}(k+1)=\phi_{m} x_{m}(k)+\Gamma_{m} u_{m}(k), \quad x_{0}=x_{m}(0) \\
y_{m}(k)=C_{m}(k)
\end{gathered}
$$

In this (8) is represented in controllable canonical state space form by considering delay and is described as

$$
\begin{gathered}
x(k+1)=\phi x(k)+\Gamma u(k)+e(k), \quad x_{0}=x(0) \\
y(k)=C x(k-w)+D u(k-w),
\end{gathered}
$$

where $\phi \in \mathfrak{R}^{n \times n}$ is state matrix, is $\Gamma \in \mathfrak{R}^{n \times 1}$ is control vector, $C \in \Re^{1 \times n}$ is output matrix, $D \in \Re^{1 \times 1}$ and $x(k)$ is state vector. The term $e(k) \in \mathfrak{R}^{n \times 1}$ represents parametric uncertainty bounded with upper limit $l_{\max }$, state $x(k) \in \mathfrak{R}^{n}$, the output $y(k) \in \mathfrak{R}^{m}$, the control $u(k) \in \mathfrak{R}^{m}$, and the disturbance $d_{d}(x, k) \in \mathfrak{R}^{m}$. Here, $w$ represents the number of delay samples and $w=t_{d} / T_{s}$, where the $T_{s}$ is the sampling period and $t_{d}$ is delay.

The discrete-time model (10) is used to generate an OMF based controller for the continuous time plant, with the assumption of zero-order command. It is assume that the system is observable and the desired estimates $\hat{x}(k)$ of the state $x(k)$ based on observation of the output $y$ alone. As in the case of the continuous observer, further, $y(k)-C \hat{x}(k)$ is estimation error. Let the observer is defined as:

$$
\begin{aligned}
\hat{x}(k+1) & =\phi \hat{x}(k)+\Gamma \mathrm{u}(\mathrm{k}) \\
& +K_{L}[y(k)-C \hat{x}(k-w)],
\end{aligned}
$$

where $K_{L}$ is the observer gain matrix [28], and $\hat{x}(k+1)$ denotes the estimate (or prediction) of $x(k+1)$ is made using measurements available at time $k$. The lumped uncertainty is:

$$
e(k)=\Delta \phi x(k)+\Delta \Gamma u(k)+d_{d}(k)
$$

The estimation error between plant and observer is defined as:

$$
\tilde{x}=x-\hat{x}
$$

Subtracting (10) from (11) gives the error difference equation: 


$$
\begin{gathered}
\tilde{x}(k+1)=\phi \tilde{x}(k-1)+\Gamma \mathrm{u}(\mathrm{k})+K_{L}[y(k)-C \tilde{x}(k-1) \\
\tilde{x}(k+1)=\left[\phi-K_{L} C\right] \tilde{x}(k-1)
\end{gathered}
$$

The observer described by Eq. (11) is known as a prediction observer, based on the fact that it predicts the state values at the next time step from past measurements. This means that control based on $\tilde{x}(k)$ does not depend on the most current measurements, which might lead to performance degradation. It is possible to construct an alternative observer formulation that provides a current estimator $\hat{x}(k)$ based on the most recent measurements of $y$. Modifying (10) to estimate:

$$
\begin{gathered}
\bar{x}(k)=\phi \hat{x}(k-1)+\Gamma \mathrm{u}(\mathrm{k}-1) \\
\hat{x}(k)=\bar{x}(k)+K_{L}[y(k)-C \bar{x}(k-1),
\end{gathered}
$$

where $\bar{x}(k)$ is the estimate based on the prediction from the previous time steps. Substitution of (16) into (15) and advancing one time-step gives

$$
\begin{aligned}
\bar{x}(k+1) & =\phi \bar{x}(k-1)+\Gamma \mathrm{u}(\mathrm{k})+K_{L}[y(k) \\
& -C \bar{x}(k-1)
\end{aligned}
$$

The error dynamics are described by:

$$
\bar{x}(k+1)=\left[\phi-K_{L} C\right] \bar{x}(k-1)
$$

The current estimate speeds up the response to unknown disturbances or measurement errors.

\section{B. Sliding Surface Selection}

The sliding surface is chosen to ensure desired behavior of surface dynamics or try the states to converged to zero. A sliding surface is selected based on Ackermann formula [26] and [19]. The observer system is given in (11) is used to calculate the equivalent control law. Define a sliding surface as:

where

$$
S(k)=\Gamma^{T} \hat{x}(k)+z(k)
$$

$$
\begin{gathered}
z(k+1)=-\Gamma^{T} \phi_{m} \hat{x}(k)-\Gamma^{T} \Gamma_{m} u_{m} \\
z(0)=-\Gamma^{T} x(0)
\end{gathered}
$$

The auxiliary variable $z$ in (20) is defined different from that given in [19]. By virtue of the choice of the initial condition on, $z(k), S(k)=0$ at $k=0$. If the control $u(k)$ can be designed ensuring sliding, then $z(k+1)=0$ implies:

$$
x_{m}(k+1)=\phi_{m} x_{m}(k)+\Gamma_{m} u_{m}
$$

This fulfills the objective of the model following approach.

\section{OMF Control Law Design Methodology}

The sliding function at $(\mathrm{k}+1)^{\text {th }}$ instant using (19) can be written as:

$$
S(k+1)=\Gamma^{T} \hat{x}(k+1)+z(k+1)
$$

$$
\begin{gathered}
S(k+1)=\Gamma^{T}\left\{\phi \hat{x}(k)+\Gamma \mathrm{u}(\mathrm{k})+K_{L}[y(k)-C \hat{x}(k-w)]\right\} \\
-\Gamma^{T} \phi_{m} \hat{x}(k)-\Gamma^{T} \Gamma_{m} u_{m}
\end{gathered}
$$

Simplifying above equation gives:

$$
\begin{aligned}
S(k+1) & =\Gamma^{T} \phi \hat{x}(k)+\Gamma^{T} \Gamma \mathrm{u}(\mathrm{k}) \\
& +\Gamma^{T} K_{L}[y(k)-C \hat{x}(k-w)] \\
& -\Gamma^{T} \phi_{m} \hat{x}(k)-\Gamma^{T} \Gamma_{m} u_{m} \\
S(k+1) & =\Gamma^{T} \hat{x}(k)\left(\phi-\phi_{m}\right)+\Gamma^{T} \Gamma \mathrm{u}(\mathrm{k}) \\
& +\Gamma^{T} K_{L}[y(k)-C \hat{x}(k-w)] \\
& -\Gamma^{T} \Gamma_{m} u_{m}
\end{aligned}
$$

From the matching conditions: $\phi-\phi_{m}=\Gamma G$ and $\Gamma_{m}=$ $\Gamma M,(24)$ becomes:

$$
\begin{aligned}
S(k+1) & =\Gamma^{T} \hat{x} \Gamma G+\Gamma^{T} \Gamma \mathrm{u}(\mathrm{k}) \\
& +\Gamma^{T} K_{L}[y(k)-C \hat{x}(k-w)] \\
& -\Gamma^{T} \Gamma M u_{m}
\end{aligned}
$$

To derive equivalent control law $u_{e q}(\mathrm{k})$, equate $S(k+1)=$ 0 , this gives:

$$
\begin{gathered}
\Gamma^{T} \hat{x} \Gamma G+\Gamma^{T} \Gamma \mathrm{u}(\mathrm{k})+\Gamma^{T} K_{L}[y(k)-C \hat{x}(k-w)] \\
-\Gamma^{T} \Gamma M u_{m}=0 \\
u_{e q}(\mathrm{k})=\left(\Gamma^{T} \Gamma\right)^{-1}\left\{-\Gamma^{T} \Gamma \hat{x} G-\Gamma^{T}\left(K_{L}[y(k)-C \hat{x}(k-w)]\right)\right. \\
\left.+\Gamma^{T} \Gamma M u_{m}\right\}
\end{gathered}
$$

Let the required control be:

$$
u(k)=u_{e q}(k)+u_{n}(k)
$$

Solving (27) for $u_{e q}(k)$

$$
\begin{gathered}
u_{e q}(\mathrm{k})=-G \hat{x}-\left(\Gamma^{T} \Gamma\right)^{-1} \Gamma^{T}\left(K_{L}[y(k)-C \hat{x}(k-w)]\right) \\
+M u_{m}
\end{gathered}
$$

Selecting

$$
\begin{gathered}
u_{e q}(k)=-G \hat{x}-\left(\Gamma^{T} \Gamma\right)^{-1} \Gamma^{T}\left(K_{L}[y(k)-C \hat{x}(k-w)]\right) \\
+M u_{m}-\left(\Gamma^{T} \Gamma\right)^{-1} K S(k)
\end{gathered}
$$

Here $K$ is a positive constant

Using (22) and (30) one can write:

$$
S(k+1)=\Gamma^{T} \Gamma u_{n}+\Gamma^{T} \Gamma e(k)-K S(k)
$$

The lumped uncertainty $e(k)$ can be estimated as given in [29]. Rewriting (31) as:

$$
e(k)=\left(\Gamma^{T} \Gamma\right)^{-1}(S(k+1)+K S(k))-u_{n}
$$

Hence lumped uncertainty $e(k)$ can be computed from (32). This cannot be done directly.

Let the estimate of uncertainty be defined as:

$$
\bar{e}(k)=\left[\left(\Gamma^{T} \Gamma\right)^{-1}(S(k+1)+K S(k))-u_{n}\right] G_{f}(s)
$$

where $G_{f}(s)$ must be proper order low pass filter with unity steady-state gain and has enough bandwidth. With such filter:

$$
\bar{e}(k) \cong e(k)
$$

This enables design of unified control as:

$$
\begin{gathered}
u_{n}=-\bar{e}(k) \\
u_{n}=-\left[\left(\Gamma^{T} \Gamma\right)^{-1}(S(k+1)+K S(k))+u_{n}\right] G_{f}(s)
\end{gathered}
$$

Finally, statement for $u_{n}$

$$
u_{n}=-\frac{\left(\Gamma^{T} \Gamma\right)^{-1}(S(k)+K S(k+1))}{T}
$$

Estimation error is:

$$
\tilde{e}(x, t)=e(k)-\bar{e}(k)
$$

\section{LYAPUNOV STABILITY CONDITIONS}

The design of OMF-SMC law ensure that the reaching condition must be satisfied when there exist uncertainty and disturbance in system. To evaluate the stability condition of proposed OMF SMC, direct Lyapunov stability analysis is used. The positive definite Lyapunov function is chosen as:

$$
\begin{gathered}
V(k)=\frac{1}{2} S^{2}(k) \\
\Delta S(k+1)=S(k+1)-S(k)
\end{gathered}
$$

Then the control law to decrease $V(k)$ is given by the following lemma.

Lemma1: If the control satisfies

$$
S(k) \Delta S(k+1)<-\frac{1}{2}(\Delta S(k+1))^{2}
$$

for

then

$$
S(k) \neq 0
$$

Proof: From (36),

$$
V(k+1)<V(k)
$$

Squaring both sides,

$$
S(k+1)=S(k)+\Delta S(k+1),
$$

$$
\begin{aligned}
S^{2}(k+1) & =S^{2}(k)+2 S(k) \Delta S(k+1) \\
& +(\Delta S(k+1))^{2}
\end{aligned}
$$

Substituting (41), (42) is derived. 
The control law to satisfy (41) should be studied. Which is the reaching condition of OMF-SMC.

\section{TUNING PARAMETER SELECTION}

Table-I: Time Domain Specifications. $M p$ is $\%$ Overshoot; $t s$ is Settling Time in Seconds at $\pm 2 \%$; No Solution.

\begin{tabular}{|c|c|c|c|c|c|}
\hline \multirow{2}{*}{ Example } & Parameter & $\begin{array}{c}\text { Proposed } \\
\text { OMF-SMC }\end{array}$ & $\begin{array}{c}\text { Khandekar } \\
\text { et. al.'s } \\
\text { DSMC }\end{array}$ & $\begin{array}{c}\text { Eker's } \\
\text { CSMC }\end{array}$ & $\begin{array}{c}\text { Wang } \\
\text { et. } \\
\text { al.'s } \\
\text { PID }\end{array}$ \\
\hline \multirow{2}{*}{1} & $t_{s}$ & 2 & 3 & 3.5 & 4.1 \\
\cline { 2 - 6 } & $M_{p}$ & 0 & 0 & 0.5 & 5.7 \\
\hline \multirow{2}{*}{2} & $t_{s}$ & 2.2 & 2.6 & 3.80 & 5.2 \\
\cline { 2 - 6 } & $M_{p}$ & 0 & 0 & 4.60 & 14.6 \\
\hline \multirow{2}{*}{3} & $t_{s}$ & 2.2 & 4 & - & - \\
\cline { 2 - 6 } & $M_{p}$ & 0 & 0 & - & - \\
\cline { 2 - 6 } & $t_{s}$ & 10 & 20.4 & - & - \\
\hline \multirow{2}{*}{5} & $M_{p}$ & 0 & 0 & - & - \\
\cline { 2 - 6 } & $t_{s}$ & 30 & 26.6 & - & - \\
\hline
\end{tabular}

The proposed OMF control law design also involves, evaluation of observer gain $K_{L}$ in equivalent control law in (30) and additional tuning term, $K$ from unified control law given in (36).

The matching conditions, $\phi-\phi_{m}=\Gamma G$ and $\Gamma_{m}=\Gamma M$ are necessary for robust asymptotic tracking performance. This tabulates matching gain matrix, $G=(\Gamma)^{-1}\left(\phi-\phi_{m}\right)$ and tuning parameter $M=(\Gamma)^{-1} \Gamma_{m}$.

The task is to place the poles of $\left|\phi-K_{L} C\right|$ arbitrarily. We choose $G=K_{L} \in \mathfrak{R}^{n \times 1}$ such that the closed-loop poles $\left|z I-\phi+K_{L} C\right|=0$, are in the desired locations. The closed-loop settling time and overshoot specifications are used to obtain the location of dominant poles, using the relation used in [24],

$$
z_{1,2}=e^{-\zeta \omega_{n} T_{s} \pm j \omega_{n} T_{s} \sqrt{1-\zeta^{2}}}
$$

where, $\zeta, \omega_{n}$ and $T_{s}$ are damping factor, natural frequency and sampling period, respectively.
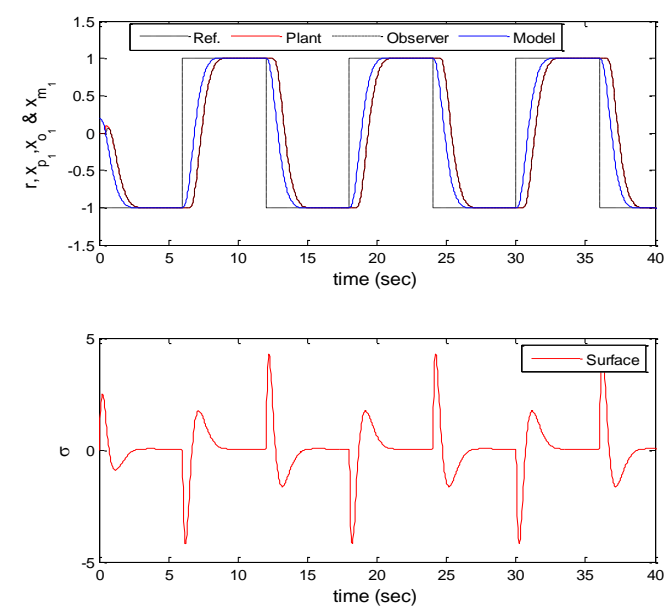

\section{SIMULATION RESULTS}

The step change responses of proposed OMF approach are shown in Fig. 1 for plant, observer and model. Five examples of higher-order linear time invariant systems such as non-oscillatory, moderate oscillatory, integrating, non-minimum phase and unstable plants are considered for simulations. These examples are from [34], [35].

\section{A. Example 1: Non-oscillatory process}

A non-oscillatory plant with continuous-time open-loop transfer function (OLTF) is:

$$
G_{p 1}(s)=\frac{1}{\left(s^{3}+11 s^{2}+35 s+25\right)} e^{-0.5 s}
$$

The equivalent DTSS model for $T s=0.1$ of $G p 1(s)$ obtained as:

$$
\begin{gathered}
\Phi=\left[\begin{array}{ccc}
0 & 1 & 0 \\
0 & 0 & 1 \\
0.3329 & -1.466 & 2.118
\end{array}\right], \quad \mathrm{H}=\left[\begin{array}{l}
0 \\
0 \\
1
\end{array}\right], \\
C=\left[\begin{array}{lll}
0.0001473 & 0.0002947 & 0.0001473
\end{array}\right]
\end{gathered}
$$

To verify the robustness and stability of OMF SMC, $20 \%$ modeling inaccuracy and bounded disturbance in $\mathrm{rad} / \mathrm{sec}$ is considered. The disturbance term is:

$$
d_{d}(x, t)=2+\cos (\pi t) .
$$

The desired stable reference model followed by plant observer is:

$$
\begin{array}{r}
\Phi_{m}=\left[\begin{array}{ccc}
0 & 1 & 0 \\
0 & 0 & 1 \\
0.3329 & -1.46 & 2.118
\end{array}\right], \quad \mathrm{H}_{m}=\left[\begin{array}{l}
0 \\
0 \\
1
\end{array}\right], \\
\mathrm{C}_{m}=\left[\begin{array}{lll}
0.0001473 & 0.0002947 & 0.0001473
\end{array}\right]
\end{array}
$$

The initial conditions for plant, observer and model are $x_{p}=$ $[0 ; 0 ; 0], x_{p o}=[0 ; 0 ; 0]$ and $x_{p m}=[0 ; 0 ; 0]$ respectively. The observer gain $K_{L}$ and matching gain $G$ is [0.2720 0.66160 .3896 ] tabulated from dominant pole placement. The other tuning parameters are considered as follows: $M=$ $1, K=0.14$. The reference input $u_{m}=25.6$ selected to validate unit step tracking performance.

The controller tuning parameters for Khandekar et. al.'s [34] Discrete Sliding Mode Control (DSMC), Eker's [36] Continuous Sliding Mode Control (CSMC) and Wang et. al.'s
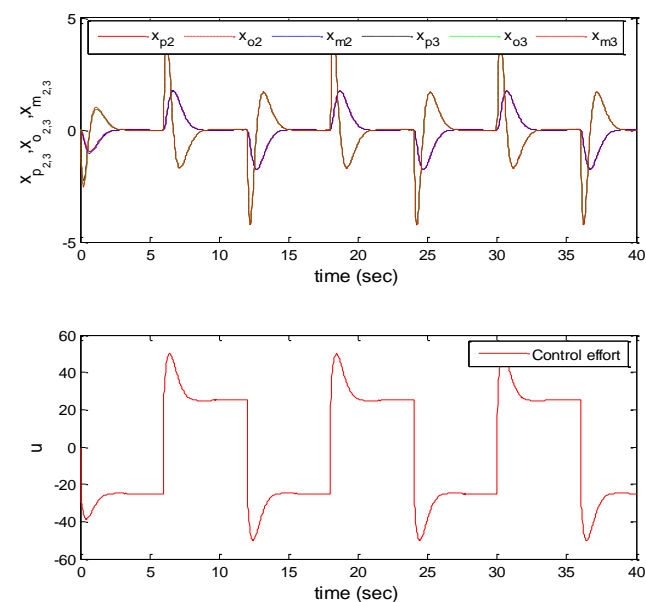

Fig. 1. Plant, observer and model states of Example 1. 
[24] Proportional-Integral-Derivative (PID) approach are considered from [23]. The parameters for Khandekar et. al.'s DSMC are $K=[0.5633-1.39370 .8304], K T=$ 30, $\alpha=30$ and $\beta=30$. The Eker's CSMC are $K_{p}=$ $2.45, K i=0.012, K_{d}=1.25$ and $K_{s w}=50$. The Wang et. al.'s PID are $K_{P}=2.45, K_{I}=0.012$ and $K_{D}=1.25$ respectively. The comparative time domain and error performance index analysis are shown in Table I-II for the simulation examples $1-5$. The unit step closed-loop responses of example 1 are shown in Fig.2 without UAD. In Fig.2(a) it is seen that plant output of proposed OMF approach has no overshoot and less settling time comparable
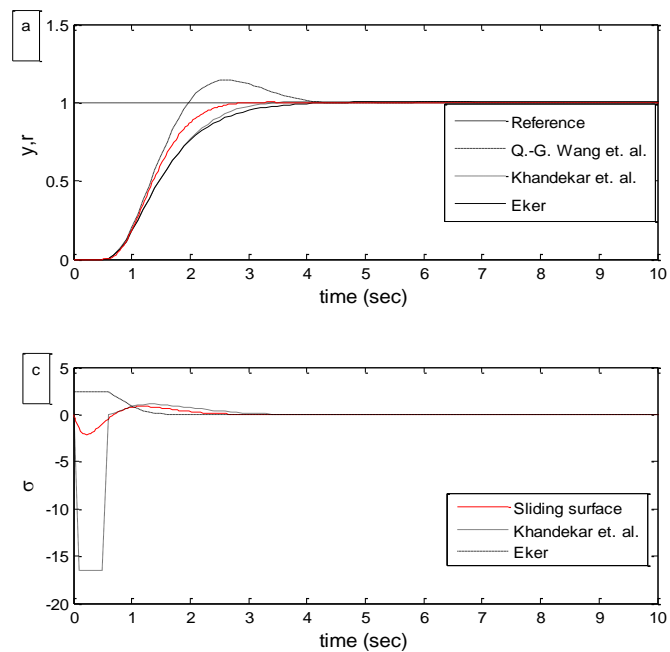

with Khandekar et. al.'s DSMC shows less overshoot, Wang et.al. PID produce large overshoot and Eker's CSMC produce large settling time. Fig.2(b) shows that plant/observer states follows the reference model accurately and states converges to zero in finite time. Fig.2(c) depicts sliding surface converges to zero smoothly, shows less oscillations compared to Khandekar et.al.'s DSMC produce large step size and small overshoot, Eker's CSMC has moderate step size. The controller's performance comparison are shown in Fig.2(d). The OMF control requires less control efforts, minimum oscillation, small step size than Khandekar et. al.'s DSMC and Eker's CSMC.
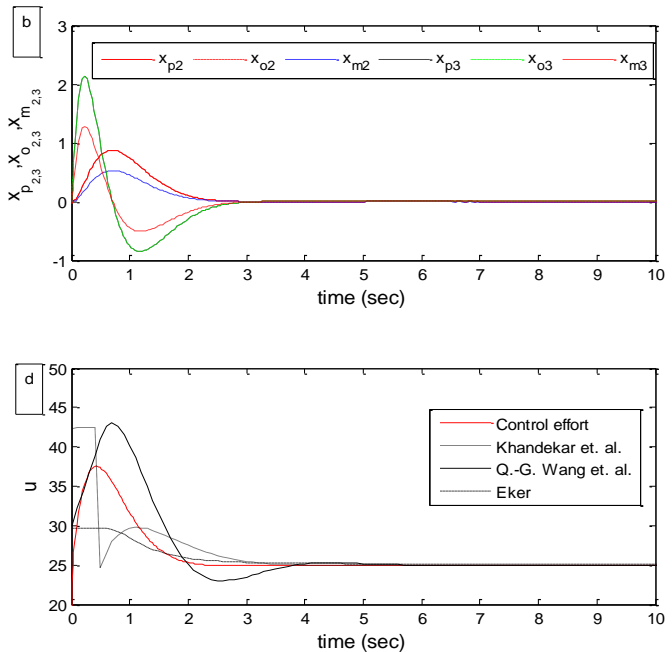

Fig. 2. (a) Output responses, (b) Plant, observer and model states, (c) Control efforts and (d) Sliding surfaces of Example 1.

Fig.3(a)-3(d) shows system performances with 20\% UAD. The tracking performance in Fig.3(a) shows similar response for Khandekar et.al's DSMC, but comparatively large overshoot, more oscillations produced by Eker's CSMC and
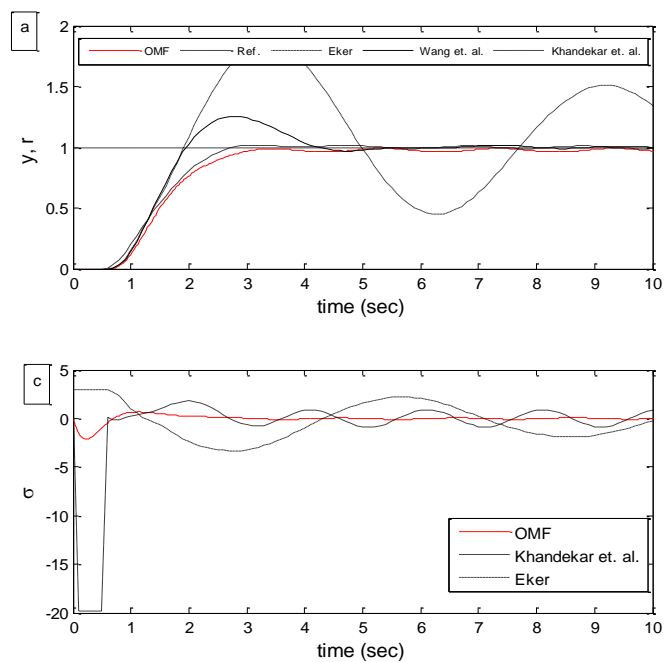

moderate overshoot shown by Wang et.al's PID. In Fig.3(d) it is seen that OMF law produce smooth response, requires less control effort compare to Khandekar et.al.'s DSMC, Eker's CSMC and Wang et.al. PID.
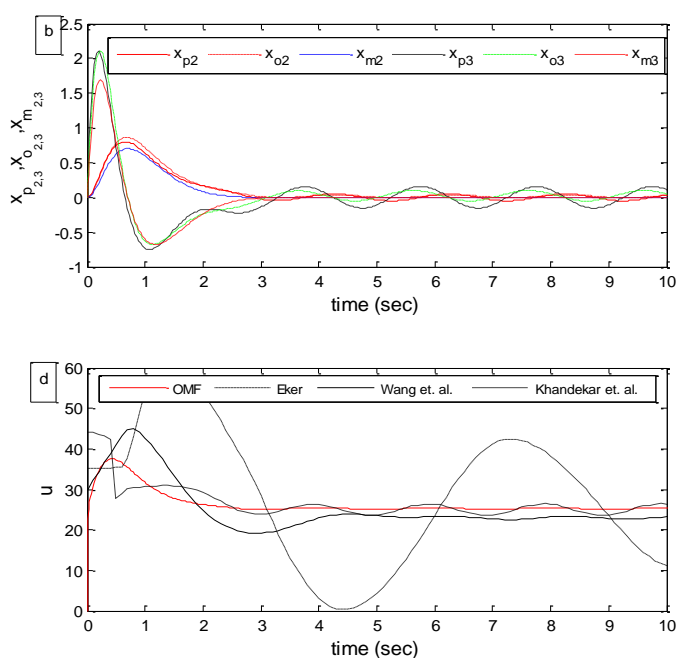

Fig. 3. (a) Output responses, (b) Plant, observer and model states, (c) Control efforts and (d) Sliding surfaces of Example 1 with $20 \%$ uncertainty.

\section{B. Example 2: Moderate-oscillatory process}

A non-oscillatory process with continuous-time open-loop transfer function (OLTF) is: 


$$
G_{p 2}(s)=\frac{1}{\left(s^{3}+5 s^{2}+9 s+9\right)} e^{-0.3 s}
$$

The equivalent DTSS model for $T_{s}=0.1$ of $G p 2(s)$ obtained as:

$$
\begin{gathered}
\Phi=\left[\begin{array}{ccc}
0 & 1 & 0 \\
0 & 0 & 1 \\
0.6065 & -2.1462 & 2.5324
\end{array}\right], \quad \mathrm{H}=\left[\begin{array}{l}
0 \\
0 \\
1
\end{array}\right], \\
C=\left[\begin{array}{llll}
0.0001953 & 0.0003905 & 0.0001953
\end{array}\right]
\end{gathered}
$$

The desired stable reference model considered as:

$$
\begin{gathered}
\Phi_{m 1}=\left[\begin{array}{ccc}
0 & 1 & 0 \\
0 & 0 & 1 \\
0.3329 & -1.466 & 2.118
\end{array}\right], \quad \mathrm{H}_{m 1}=\left[\begin{array}{l}
0 \\
0 \\
1
\end{array}\right], \\
\mathrm{C}_{m 1}=\left[\begin{array}{lll}
0.0001953 & 0.0003905 & 0.0001953
\end{array}\right]
\end{gathered}
$$

The tuning parameters are computed as follows for example
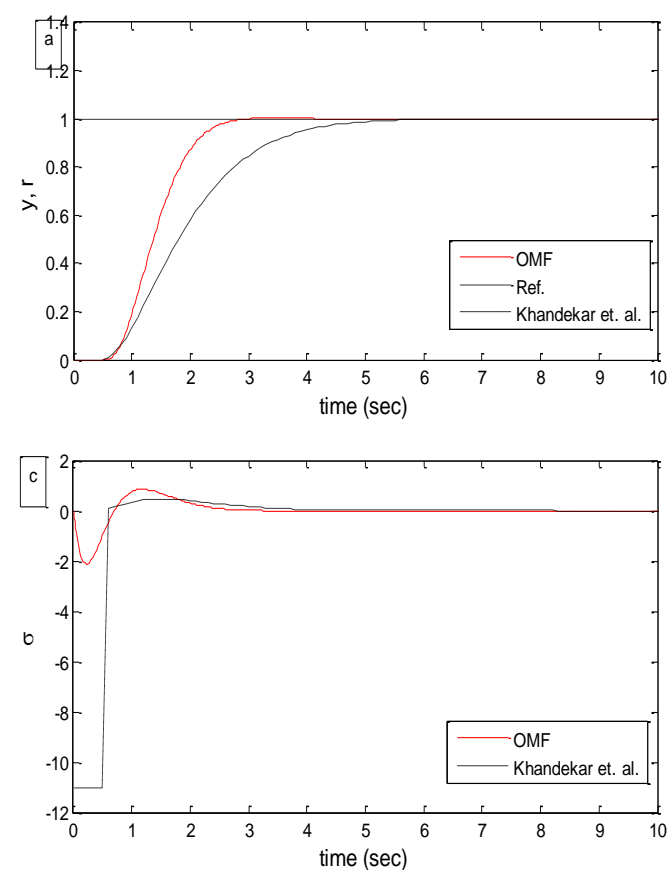

2: $G=K_{L}=[0.5633-1.39370 .8304], \quad M=0.480$, $K=0.22, \quad u_{m}=19.4$. The tuning parameters for Khandekar et. al. DSMC, Eker's CSMC and Wang et. al.'s PID are used from [34].

In Fig.4(a), it can be seen that plant output reaches to unit step in less time compared to Khandekar et. al's DSMC. Similarly, Eker's CSMC and Wang et. al.'s PID produces quite oscillations. From Fig.4(d), it is seen that the proposed controller produces smooth response with less and smooth control effort while the response produced by Khandekar et. al's DSMC results in large control effort. Eker's CSMC and Wang et. al.'s PID controller responses seen are satisfactory.
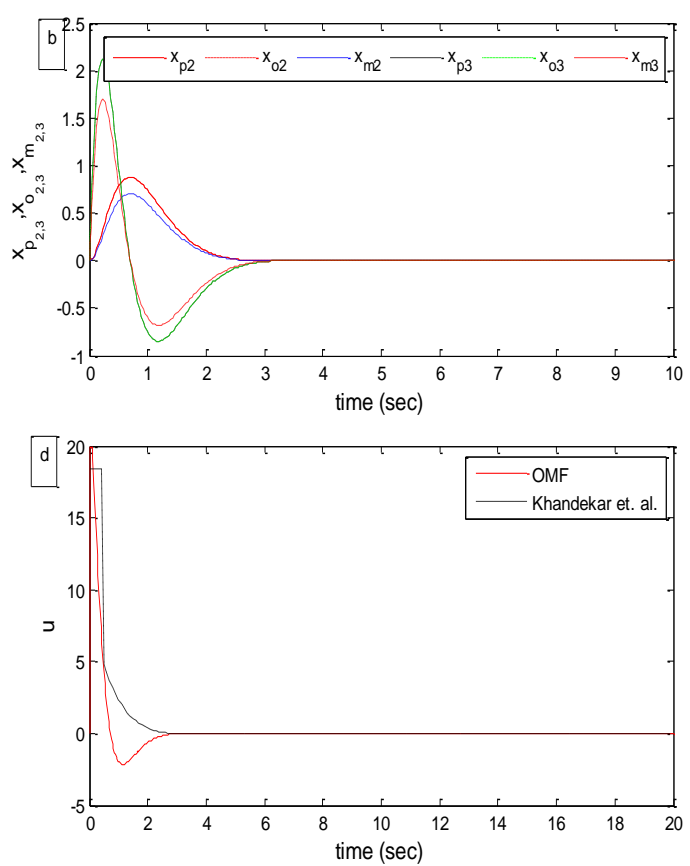

Fig. 4. (a) Output responses, (b) Plant, observer and model states, (c) Control efforts and (d) Sliding surfaces of Example 2.

\section{Example 3: Integrating process}

Consider an integrating process with OLTF [25]

$$
G_{p 3}(s)=\frac{1}{s(s+1)(s+3)} e^{-0.5 s}
$$

The equivalent DTSS model for $T_{s}=0.1$ of $G_{p 3}(s)$ obtained as:

$$
\begin{gathered}
\Phi=\left[\begin{array}{ccc}
0 & 1 & 0 \\
0 & 0 & 1 \\
0.5488 & -2.0602 & 2.5114
\end{array}\right], H=\left[\begin{array}{l}
0 \\
0 \\
1
\end{array}\right], \\
C=\left[\begin{array}{lll}
0.0001873 & 0.0003746 & 0.00018473
\end{array}\right]
\end{gathered}
$$

The desired stable reference model considered as:

$$
\begin{aligned}
\Phi_{m 3} & =\left[\begin{array}{ccc}
0 & 1 & 0 \\
0 & 0 & 1 \\
0.3329 & -1.466 & 2.118
\end{array}\right], \mathrm{H}_{m 3}=\left[\begin{array}{l}
0 \\
0 \\
1
\end{array}\right], \\
\mathrm{C}_{m 3} & =\left[\begin{array}{lll}
0.0001873 & 0.0003746 & 0.00018473
\end{array}\right]
\end{aligned}
$$

The tuning parameters are computed as follows for example:
$G=K_{L}=[0.4669-1.06870 .6018], M=0.001, K=$ $0.120, u_{m}=20.0$. The tuning parameters for Khandekar et. al. DSMC are considered from [34].

The system performances of example 3 are shown in Fig.5 are without UAD. In Fig.5(a) plant output are compared with Khandekar et. al's DSMC [34] and there is no solution for Eker's CSMC and Wang et. al.'s PID. It is seen that proposed OMF method results in an better response with no overshoot and less settling time. Khandekar et. al.'s DSMC has large settling time. Fig.5(b) shows OMF plant, observer and model states responses converges to zero in less time. Fig.5(c) compares sliding surface responses. The proposed OMF surface converges to zero smoothly with less oscillations compared to Khandekar et.al.'s DSMC has large step size. Fig.5(d) further shows a controller performances. The proposed method requires similar control effort compared with Khandekar et.al's DSMC [34]. 

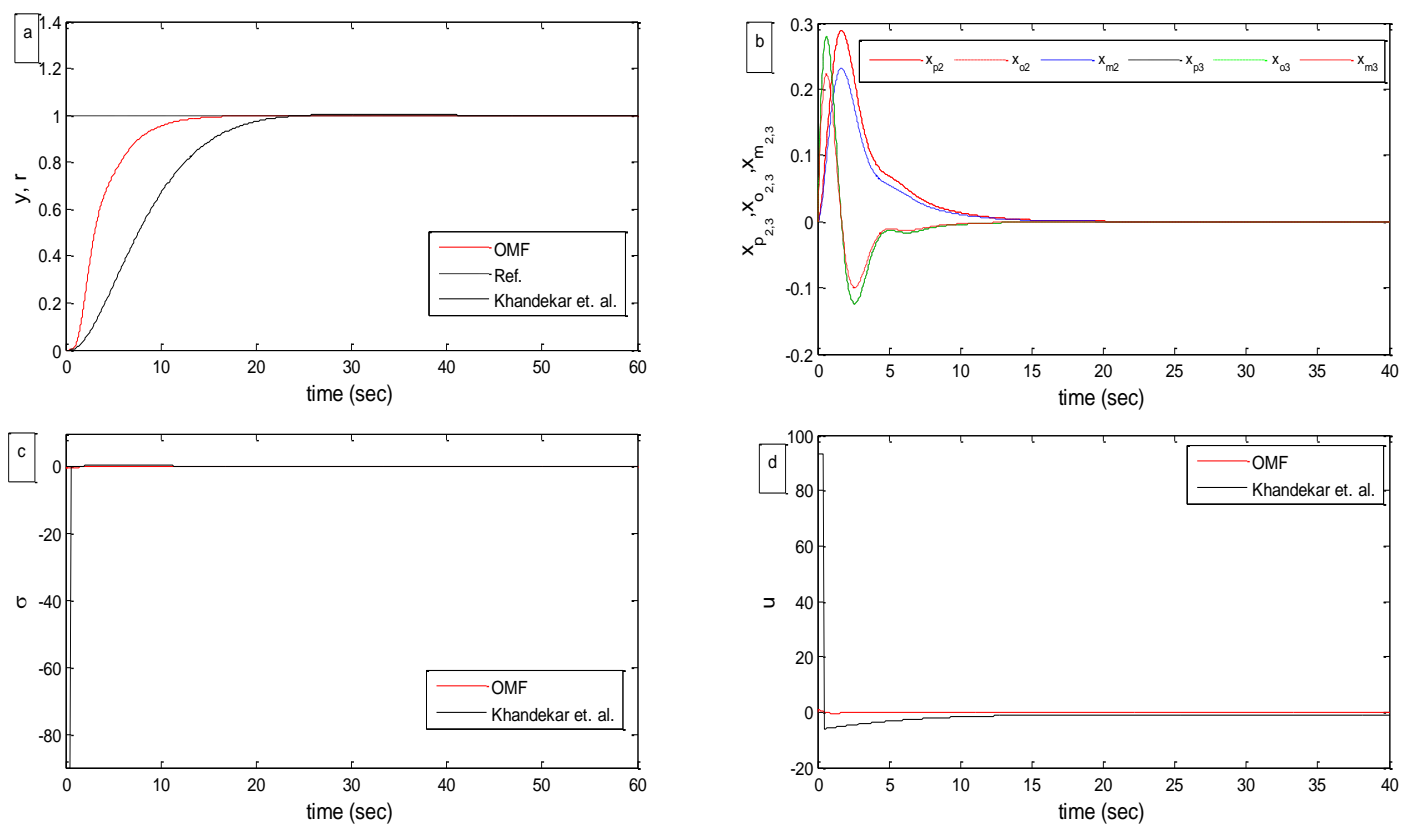

Fig. 5. (a) Output responses, (b) Plant, observer and model states, (c) Control efforts and (d) Sliding surfaces of Example 3.

\section{Example 4: Unstable process}

A non-oscillatory process with continuous-time open-loop transfer function (OLTF) is:

$$
G_{p 4}(s)=\frac{1}{(1-2 s)(1+5 s)(1+20 s)} e^{-0.5 s}
$$

The equivalent DTSS model for $T_{s}=0.1$ of $G p 4(s)$ obtained as:
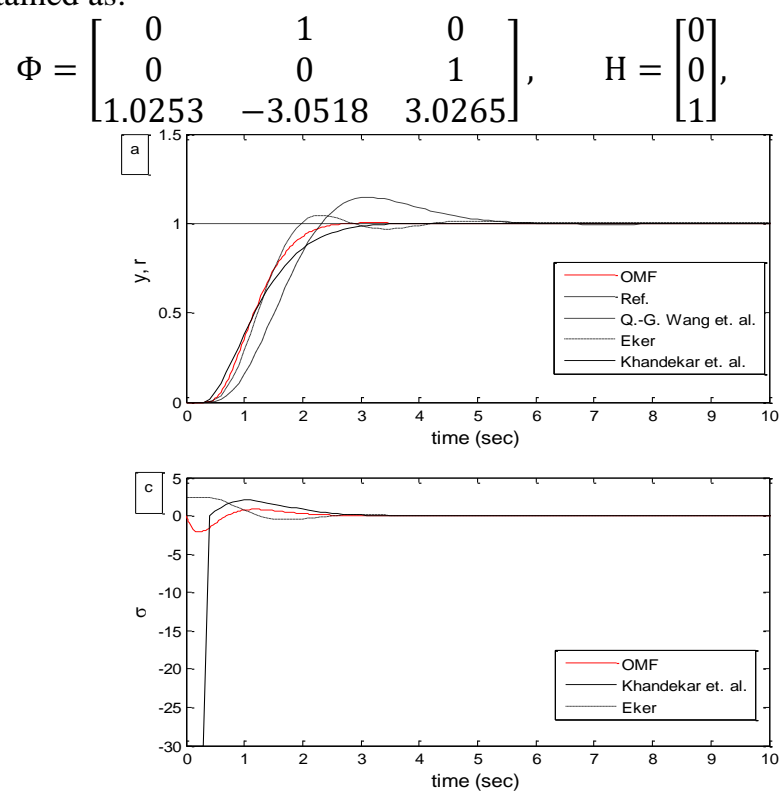

$C=[-0.000001266-0.000002532-0.000001266]$

The reference model that is to be followed is selected as:

$$
\Phi_{m 4}=\left[\begin{array}{ccc}
0 & 1 & 0 \\
0 & 0 & 1 \\
-30 & -40 & -15
\end{array}\right], \quad \mathrm{H}_{m 4}=\left[\begin{array}{c}
0 \\
0 \\
30
\end{array}\right],
$$$$
\mathrm{C}_{m 4}=\left[\begin{array}{lll}
-0.0001473 & -0.0002947 & -0.0001473
\end{array}\right]
$$

The tuning parameters are computed as follows for example4: $\quad G=K L=[0.9292-1.89530 .9661, M=$ $1.730, K=0.0153, u_{m}=-25.1$. The tu ning parameters for Khandekar et. al. DSMC are used from [34].
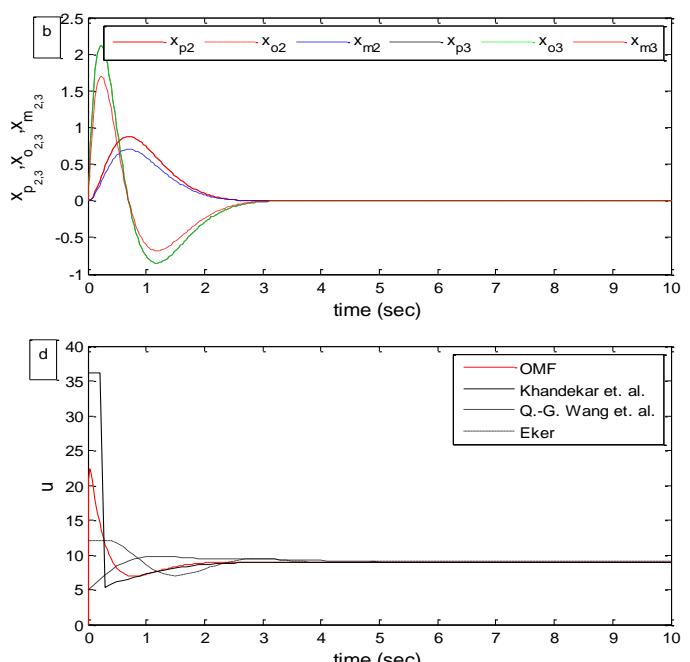

Fig. 6. (a) Output responses, (b) Plant, observer and model states, (c) Control efforts and (d) Sliding surfaces of Example 4.

The closed-loop performances of example 4 without UAD are shown in Fig.6. In Fig.5(a) plant output of proposed OMF method has less settling time compared with Khandekar et. al's DSMC [34] has large settling time. There is no solution for Eker's CSMC and Wang et. al.'s PID. Fig.6(b) shows plant, observer and model states responses of OMF method. Fig.6(c) shows sliding surface responses. The proposed OMF surface converges to zero smoothly with less oscillations compared to Khandekar et.al.'s DSMC has large step size. Fig.6(d) further shows a controller performances. The proposed method requires small control effort compared with Khandekar et.al's DSMC [34] requires large step size. 


\section{E. Example 5: Non-minimum phase process}

A non-oscillatory process with continuous-time open-loop transfer function (OLTF) is:

$$
G_{p 5}(s)=\frac{(1-4 s)}{(1+2 s)(1+5 s)(1+20 s)} e^{-0.1 s}
$$
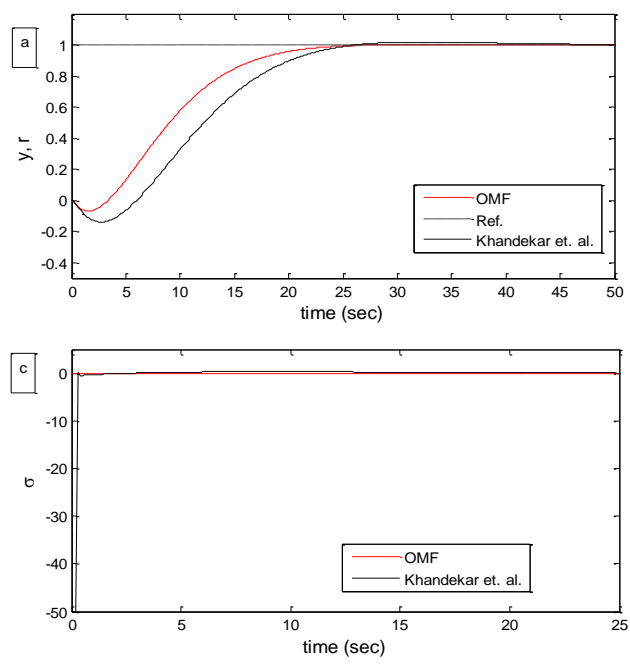

The equivalent DTSS model for $T_{s}=0.1$ of $G p 5(s)$ obtained

$$
\begin{aligned}
& \Phi=\left[\begin{array}{ccc}
0 & 1 & 0 \\
0 & 0 & 1 \\
0.9277 & -2.8542 & 2.9264
\end{array}\right], \quad \mathrm{H}=\left[\begin{array}{l}
0 \\
0 \\
1
\end{array}\right], \\
& C=\left[\begin{array}{lll}
0.00009754 & 0.00000241
\end{array}\right]
\end{aligned}
$$
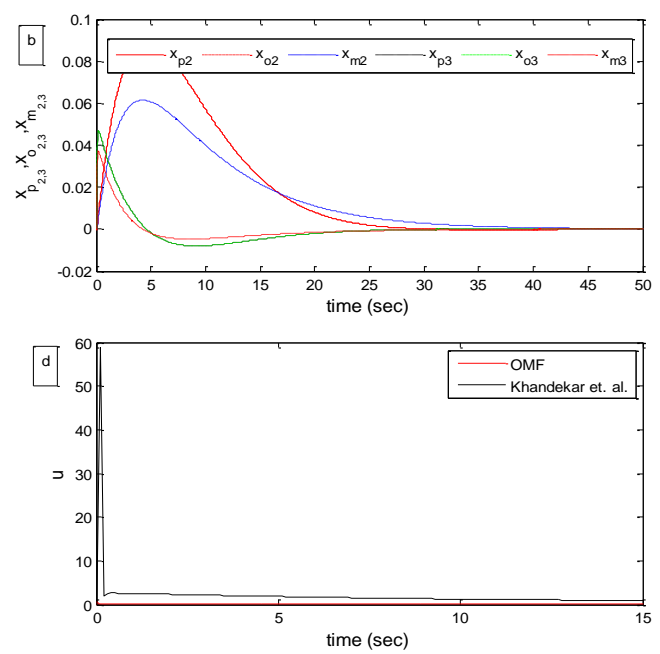

Fig. 7. (a) Output responses, (b) Plant, observer and model states, (c) Control efforts and (d) Sliding surfaces of Example 5.

The desired stable reference model considered as:

$$
\begin{gathered}
\Phi_{m 5}=\left[\begin{array}{ccc}
0 & 1 & 0 \\
0 & 0 & 1 \\
0.3329 & -1.466 & 2.118
\end{array}\right], \quad \mathrm{H}_{m 5}=\left[\begin{array}{l}
0 \\
0 \\
1
\end{array}\right], \\
\mathrm{C}_{m 5}=\left[\begin{array}{lll}
0.0001473 & 0.0002947 & 0.0001473
\end{array}\right]
\end{gathered}
$$

The tuning parameters are computed as follows for example5: $G=K_{L}=[0.8994-$ $1.83600 .9366], M 2.0400, K=0.01, u m=25.5$. The tuning parameters for Khandekar et. al. DSMC are used from [34].

The comparative performances of example 5 without UAD are shown in Fig.7. In Fig.7(a) plant output of proposed OMF method produce no overshoot and minimum settling time compared to Khandekar et. al's DSMC [34] shows small over shoot. There is no solution for Eker's CSMC and Wang et. al.'s PID. Fig.7(b) shows plant, observer and model states responses of OMF method. Fig.7(c) shows sliding surface responses. The proposed OMF surface converges to zero smoothly with less oscillations compared to Khandekar et.al.'s DSMC has large step size. Fig.7(d) further shows a controller performances. The proposed method requires

\begin{tabular}{|c|c|c|c|c|c|c|c|c|c|c|c|c|c|c|c|c|}
\hline \multirow{2}{*}{ Ex. } & \multicolumn{4}{|c|}{ OMF-SMC } & \multicolumn{4}{|c|}{ Khandekar et. al.'s DSMC } & \multicolumn{4}{|c|}{ Eker's CSMC } & \multicolumn{4}{|c|}{ Wang et. al.'s PID } \\
\hline & ISE & ITSE & IAE & ITAE & ISE & ITSE & IAE & ITAE & ISE & ITSE & IAE & ITAE & ISE & ITSE & IAE & ITAE \\
\hline 1 & 31.8 & 5.44 & 39.8 & 16.7 & 12.8 & 8.6 & 16.3 & 14.6 & 12.9 & 10.02 & 17.6 & 27.4 & 12.6 & 7.68 & 15.9 & 15.06 \\
\hline 2 & 31.8 & 5.44 & 39.8 & 16.7 & 10.39 & 4.89 & 13.6 & 10.4 & 10.75 & 5.63 & 14.35 & 21.5 & 13.9 & 11.29 & 18.11 & 21.9 \\
\hline 3 & 31.8 & 5.44 & 39.8 & 16.7 & 15.1 & 16.9 & 20.5 & 25.06 & - & - & - & - & - & - & - & - \\
\hline 4 & 72.1 & 272.5 & 114.8 & 310.01 & 58.3 & 1368.6 & 85.9 & 494.2 & - & - & - & - & - & - & - & - \\
\hline 5 & 237.1 & 6235.5 & 312.5 & 1852.4 & 122.8 & 11989 & 177.3 & 2340.2 & - & - & - & - & - & - & - & - \\
\hline
\end{tabular}
small control effort compared with Khandekar et.al's DSMC [34] requires large step size.

Table- II: Error Performance Index. ISE is Integral Square Error; ITSE is Integral Time Square Error; IAE is Integral Absolute Error; IT AE is Integral Time Absolute Error; - No Solution

\section{CONCLUSION}

In this study, OMF-SMC is presented to improve the performance of discrete linear control systems. The approach is investigated on different linear systems. Simulation results shown that the proposed control approach work well and shows robustness to changes in uncertainty and disturbance. Based on simulation results and performance analysis using peak overshoot, settling time and error performance indices, it can be concluded that performance of higher order linear time delay systems is improved with proposed controller compared with PID controller of Wang et. al, CSMC of Eker's and DSMC of Khandekar et. al. Thus, the proposed OMF-SMC presented in this paper is effective and its use can be extended to experimental processes. 


\section{REFERENCES}

1. V. I. Utkin, "Variable structure systems with sliding modes," IEEE Trans. Automatic Control, vol. 22, no. 2, Apr. 1977, pp. 212-222.

2. Q-G Wang, T-H Lee, H-W Fung, B. Qiang, and Y. Zhang, "PID tuning for improved performance," IEEE Transactions on Control System Technology, vol. 7, no.4, Jul. 1999, pp. 457-65.

3. C-T Chen and S-T Peng, "Design of a sliding-mode control system for chemical proces," Journal of Process Control, vol. 15, no. 5, 2005, pp. $515-530$.

4. K. Furuta, "Sliding-mode control of a discrete system," Syst. Contr. Lett., vol. 14, no.2, 1990, pp. 145-152.

5. W. Gao, Y. Wang, and A. Homaifa, "Discrete-time variable structure control systems," IEEE Trans. Ind. Electron., vol. 42, no. 2, Apr. 1995, pp. 117-122.

6. M. Mihoub, A. S. Nouri, and R.B. Abdennour, "Real-time application of discrete second order sliding-mode control to a chemical reactor," Control Engineering Practice, vol. 17, no. 9, Sep. 2009, pp. 1089-1095.

7. Q.-C. Zhong and D. Rees, "Control of uncertain LTI systems based on an uncertainty and disturbance estimator," Journal of Dynamic Systems, Measurement, and Control, vol. 126, 2004, pp. 905-910.

8. S. B. Phadke and S.E. Talole, "Sliding Mode and Inertial Delay Control Based Missile Guidance," IEEE Trans on Aerospace and Electronic Systems, vol. 48, no. 4, Oct. 2012, pp. 3331-3346.

9. V. S. Deshpande and S.B. Phadke, "Control of uncertain nonlinear systems using an uncertainty and disturbance estimator," Trans ASME, J. Dynamic System Meas. And Control, vol. 134, no. 2, 2012, p. 024501.

10. V. S. Deshpande, P.D. Shendge and S.B. Phadke, "Active suspension system for vehicles based on a sliding mode control in combination with inertial delay control," Proceedings of the Inst. of Mechanical Engineers, Part of D: Journal of Automobile Engineering, vol. 227, no. 5, Nov. 2012, pp. 675-690.

11. P. V. Suryawanshi, P.D. Shendge, and S.B.Phadke, "Robust Sliding mode control for a class of nonlinear systems using inertial delay control," Nonlinear Dynamics, vol. 78, no. 3, Jul. 2014, pp. 1921-1932.

12. R. P. Vieira, C. C. Gastaldini, R. Z. Azzolin, and H. A. Grundling, "Sensorless sliding-mode rotor speed observer of induction machines based on magnetizing current estimation," IEEE Transactions on Industrial Electronics, vol. 61, no. 9, Sep. 2014, pp. 4573-4582.

13. T. Bernardes, V. M. Foletto, H. A. Grundling, and H. Pinheiro, "Discrete Time Sliding mode Observer for Sensorless Vector Control of Permanent Magnet Synchronous Machine," IEEE Transactions on Industrial Electronics, vol. 61, no.4, Apr. 2014, pp. 1679-1691.

14. R. P. Vieira, C. C. Gastaldini, R. Z. Azzolin, and H. A. Gründling, "Discrete-time sliding-mode speed observer for sensorless control of induction motor drives," IET Electric Power Applications, vol. 6, no.9, 2012, pp. 681-688.

15. V. Acary, B. Brogliato, and Y. V. Orlov, "Comments on Chattering Free Digital Sliding Mode Control With State Observer and Disturbance Rejection," IEEE Transactions on Automatic Control, vol 61, no. 11, Nov. 2016, pp. 3707-3707.

16. J. Zhang, G. Feng, and Y. Xia, "Design of Estimator Based Sliding Mode Output Feedback Controllers for Discrete-Time Systems," IEEE Transactions on Industrial Electronics, vol. 61, no.5, May 2014, pp. 2432-2440.

17. H. Yang, Y. Xia, and P. Shi, "Observer based sliding mode control for a class of discrete systems via delta operator approach," Journal of Franklin Institute, vol. 347, no.7, Sep. 2010, pp. 1199-1213.

18. C. L. Baratieri and H. Pinheiro, "New variable gain super-twisting sliding-mode observer for sensorless vector control of non-sinusoidal back-EMF PMSM," Control Engineering Practice, vol. 52, Jul. 2016, pp. 59-69.

19. H. Niu, Q. Lan, Y. Liu, and H. Xu, "A continuous integral terminal sliding mode control approach for a class of uncertain nonlinear systems," Measurement and Control, vol. 52, no. 5-6, Apr. 2019, pp. 720-728.

20. H. Hou, X. Yu, L. Xu, K. A. Rsetam, and Z. Cao, "Finite-Time Continuous Terminal Sliding Mode Control of Servo Motor Systems," IEEE Transactions on Industrial Electronics, 2019, pp. 1-1.

21. M. -C. Pai, "Adaptive super-twisting terminal sliding mode control for nonlinear systems with multiple inputs," International Journal of Dynamics and Control, Nov. 2019.

22. R. Hendel, F. Khaber, and N. Essounbouli, "Adaptive high order sliding mode controller/observer based terminal sliding mode for MIMO uncertain nonlinear system," International Journal of Control, Mar. 2019, pp. 1-21.

23. A. M. Singh and Q. P. Ha, "Fast Terminal Sliding Control Application for Second-order Underactuated Systems," International Journal of
Control, Automation and Systems, vol. 17, no. 8, May 2019, pp. 1884-1898.

24. S. Chen, W. Liu, and H. Huang, "Nonsingular Fast Terminal Sliding Mode Tracking Control for a Class of Uncertain Nonlinear Systems," Journal of Control Science and Engineering, vol. 2019, May 2019, pp. $1-17$.

25. M. Boukattaya, N. Mezghani, and T. Damak, "Adaptive nonsingular fast terminal sliding-mode control for the tracking problem of uncertain dynamical systems," ISA Transactions, vol. 77, June 2018 pp. $1-19$

26. M. Labbadi and M. Cherkaoui, "Robust adaptive nonsingular fast terminal sliding-mode tracking control for an uncertain quadrotor UAV subjected to disturbances," ISA Transactions, Nov. 2019.

27. R. Qi, W. Su, and Y. Meng, "Fault-Tolerant Attitude Controller Design for Deep Space Probe Via Adaptive Fast Terminal Sliding Mode Control," Journal of Dynamic Systems, Measurement, and Control, vol. 141, no. 9, May 2019.

28. H. Rabiee, M. Ataei, and M. Ekramian, "Continuous nonsingular terminal sliding mode control based on adaptive sliding mode disturbance observer for uncertain nonlinear systems," Automatica, vol. 109, Nov. 2019, p. 108515.

29. K. Eliker and W. Zhang, "Finite-time Adaptive Integral Backstepping Fast Terminal Sliding Mode Control Application on Quadrotor UAV," International Journal of Control, Automation and Systems, Sep. 2019.

30. S. E. Talole and S.B. Phadke, "Robust input-output linearization using uncertainty and disturbance estimation," International Journal of Control, vol. 82, no. 10, Aug. 2009, pp. 1794-1803.

31. J. E. Ackerman, Pole Placement Control, Control Systems, Robotics and Automation, vol -III.

32. D. G. Luenberger, "Observing the states of a linear systems," IEEE Transactions on Military Electronics, vol. 8, no. 2, 1964, pp. 74-80.

33. C. Edwards and S. K. Spurgeon, Sliding-mode Control: Theory and Applications, CRC Press, Aug. 1998.

34. A. A. Khandekar and B. M. Patre, "Discrete sliding mode control for robust tracking of time-delay systems," Systems Science and Control Engineering, vol. 2, no. 1, Apr. 2014, pp. 457-464.

35. Y. P. Patil, H. G. Patel, and B. B. Musmade, "Robust control design using discrete sliding mode control for uncertain systems," International Conference on Intelligent Systems and Control (ISCO), Jan. 2017.

36. I. Eker, "Second order sliding mode control with the experimental application," ISA Transactions, vol. 49, no. 3, Jul. 2010, pp. 394-405.

\section{AUTHORS PROFILE}

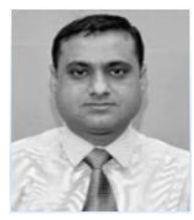

Y. P. Patil received $M$. Tech. degree in Instrumentation and Control Engineering in the year 2010 at Government College of Engineering, Pune, India. He has presented several conference papers at various international conferences. His research topic is sliding mode control design for process control applications. He is life member of Indian Society for Technical Education (ISTE) and annual member of Automatic Control and Dynamical Optimization Society (ACDOS).

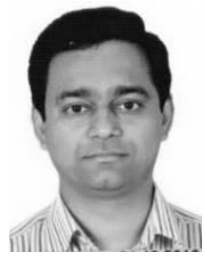

H. G. Patel received the Ph.D. degree from Electrical Engineering Department, SVNIT University, Surat. He specializes in control systems, nonlinear stochastic, sliding mode control. He has published several research papers in various international journals and presented various papers in international conferences. He is a co-author of book chapter published by Sycio Science publisher. 